\title{
Hexagonal patterns in laser light
}

SIR - In 1969, Schwarz and Hora reported an effect ${ }^{1-3}$ in which low-power laser light scattered through quartz produced hexagonal patterns ( $a$ in the figure). This effect has defied explanation and reproduction since its discovery and

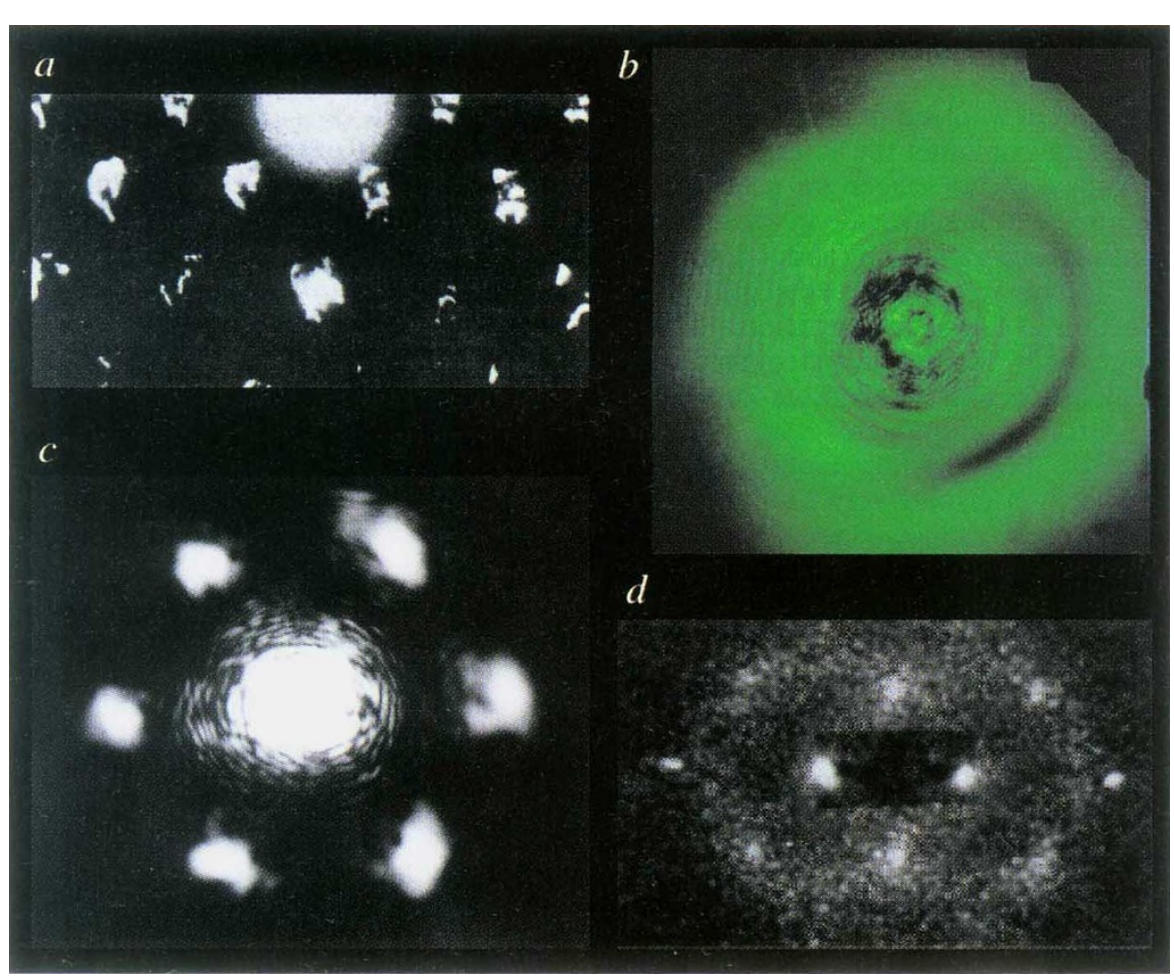

a, Intermediate-field pattern of argon-ion laser light in quartz in the original Schwarz-Hora experiment $^{1} ; b$, far-field pattern in PMN crystals (present work: $1 / 30$-s exposure; dark ring is at $1.0^{\circ}$ ); $c$, potassium niobate ${ }^{5}$ pattern; $d$, colloidal suspensions ${ }^{14}$.

has often been regarded with scepticism, although recently Honda discovered striking hexagonal patterns in the laser light scattered from $\mathrm{KNbO}_{3}$ (ref. 4). Here we report a similar, reproducible effect: low-intensity laser light scattered through crystals gives rise to hexagonal intensity patterns. We show that the phenomenon arises from thermal focusing in photorefractive media.

1. Schwarz, H. \& Hora, H. Appl. Phys. Lett. 15, 349-351

2. Harris, R. L. \& Smith, R. F. Nature 225, 502 (1970).

3. Schwarz, H. Nature 225, 1173 (1970).

4. Honda T. Opt. Lett. 20, 851-853 (1995).

5. Chen, T., Scott, J. F. \& Phillipson, P. E. Integ. Ferroelec. 5. 1-12 (1994).

6. Banerjee, P. P. et al. Opt. Lett. 20, 10-12 (1995)

7. Grynberg, G. Opt. Commun. 66, 321-324 (1988)

8. Firth, W. J. J. Mod. Opt. 37, 151-153 (1990).

9. Nelson, D. R. \& Halperin, B. I. Phys. Rev. B 19, 2457-2484 (1979)

10. Mermin, N. D. Phys. Rev. 176, 250-254 (1968).

11. Kosterlitz, J. M. \& Thouless, D. J. J. Phys. C 6 , 1181-1203 (1973).

12. Scott, J. F., Sargent, M. \& Cantrell, C. D. Opt. Commun. 15, 13-15 (1975)

13. Macdonald R. \& Eichler H. J. Opt. Commun. 89 289-295 (1992)

14. Chowdhury, A., Ackerson, B. J. \& Clark, N. A. Phys. Rev. Lett. 55, 833-836 (1985).
We have carried out extensive studies ${ }^{5}$ in ferroelectric crystals with thermal focusing nonlinearities (in the simplest case, as with fluids, the density and hence index of refraction decreases in heated regions due to thermal expansion). We

observe ( $b$ in the figure) far-field optical patterns in $\mathrm{PbMg}_{1 / 3} \mathrm{Nb}_{2 / 3} \mathrm{O}_{3}(\mathrm{PMN})$, that contain interference rings with intensity distributed with a 6-fold symmetry around the beam direction. There is a threshold for this effect.

We find that these patterns do not rotate when the sample is rotated, ruling out crystallographic-diffraction explanations. Banerjee et al. report ${ }^{6}$ the complementary effect of rotation of the images with stationary geometry, which we believe to be due to photorefraction with transverse thermal instabilities?. Firth's relationship ${ }^{8}$ determines the wavevector $K$ of the transverse instability: $K^{2} L=3 \pi n k / 2$, where $L$ is the path length; $n$, the index of refraction (2.4); and $k$, the wavevector of the laser $\left(19,436 \mathrm{~cm}^{-1}\right)$. Hence $K$ is $2,850 \mathrm{~cm}^{-1}$ and scattering angle $\Theta=\tan ^{-1}(K / k)=0.45^{\circ}$, about midway between the value $1.0^{\circ}$ observed in $\mathrm{KNbO}_{3}$ and $0.20^{\circ}$ measured in PMN and comparable to that in the Schwarz-Hora effect.

Immediately after illumination, a circular ring is observed in the far-field pattern of several ferroelectric oxides ${ }^{5}$, purely a thermal-lens effect arising from interference between light scattered from the centre and edges of the illuminated region. If the laser power is sufficient that the maximum thermal-lens diffraction angle exceeds that for pattern formation, $\Theta$, then within a few seconds the ring coalesces into six spots. This shows that thermal defocusing provides the transverse nonlinearity necessary for pattern formation, although the observability of the pattern is intrinsically a photorefractive effect, caused by a change in refractive index due to the electric field of the light.

These phenomena can be described elegantly using the theory of two-dimensional melting. As noted by others ${ }^{9,10}$, Kosterlitz-Thouless theory ${ }^{11}$ predicts a two-step evolution of diffraction patterns: the second step is from a circular Debye-Scherrer ring to a hexatic phase with 6-fold symmetry and angularly broadened spots. In this interpretation the unilluminated specimens contain a random collection of charged defects. The standing light wave causes them to order ('freeze'), and spontaneous symmetry breaking in two dimensions causes the 'Debye-Scherrer' circular diffraction pattern to evolve into a hexagonal pattern. (We see this effect only in singlecrystal PMN, not in ceramics where diffusion of defects through grain boundaries to produce the requisite holographic gratings is prevented.) At high laser intensity, pattern assembly becomes continuous. This satisfies the tricritical nature $^{12}$ of other nonequilibrium 'phase transitions'.

Schwarz and Hora mentioned that the far-field patterns resembled the electron diffraction patterns in the target crystals; but our explanation offered here suggests that this resemblance was misleading ${ }^{13}-$ hexagonal patterns would still have resulted if cubic lattices ${ }^{14}$ had been used as targets.

J. F. Scott

Faculty of Science,

University of New South Wales,

Sydney 2052,

Australia

\section{R. A. O'Sullivan}

Department of Applied Physics,

Royal Melbourne Institute of Technology,

Melbourne 3001,

Australia

\section{Scientific Correspondence}

Scientific Correspondence is intended to provide a forum in which readers may raise points of a scientific character. Priority will be given to letters of fewer than 500 words and five references. 